\title{
Observation of interference fringes in Autler-Townes line shapes
}

\author{
Steven R. Wilkinson* and Arlee V. Smith \\ Laser, Optics, and Remote Sensing, Department 1128, Sandia National Laboratories, Albuquerque, New Mexico 87185-1423 \\ and Department of Physics and Astronomy, University of New Mexico, Albuquerque, New Mexico 87135 \\ Marlan O. Scully and Ed Fry \\ Department of Physics, Texas A\&M University, College Station, Texas 77834
}

(Received 30 May 1995)

\begin{abstract}
We observe Ramsey fringes in the absorption line shape for a weak optical field probing the transition between an unperturbed quantum state and one that is dynamically Stark shifted by a strong pump field.
\end{abstract}

PACS number(s): 32.30.Jc, 32.70.Jz, 42.50.Hz, 42.50.Md

There have been several observations of quantum interference effects due to two time-separated interactions. The first, by Ramsey [1], used an atomic beam with two spatially separated $\mathrm{rf}$ interaction regions. Atoms passing through the first region were placed in a coherent superposition of quantum states. In the second interaction region, these atoms either emitted or absorbed radiation depending on the relative phase of the residual coherence and the second rf field. As the rf field was tuned through a resonance, interference fringes appeared in the absorption spectrum with a period equal to one-half the flight time between the interaction regions. These fringes are commonly known as Ramsey fringes. They have also been experimentally observed in the optical region [2] using Doppler-free two-photon transitions in sodium atoms excited with two time-separated laser pulses.

Another example of quantum interference due to timeseparated interactions arises in nonresonant excitation of atoms during a collision. Here the collision alters the separation of two molecular energy levels so that light tuned near the transition can be resonant at two different times [3] during the collision, once when the interatom separation is decreasing and once when it is increasing. The interference can be constructive or destructive depending on the evolution of the energy levels between the two interactions.

In a third example, interference has been seen in multiphoton absorption in the microwave [4] and optical regimes [5]. The ac Stark shifts created by the pulsed microwave or optical field move the transition into and out of multiphoton resonance during the pulse. Thus the transition is resonant with the driving field at two times during the pulse, with a time separation depending on the pulse intensity and the detuning from multiphoton resonance. For a fixed pulse intensity, fringes appear in the excitation spectrum. Alternatively, for a fixed driving microwave or optical frequency, fringes appear as a function of pulse intensity.

We report here observation of another separated-time interference effect in a one-photon transition between an unperturbed upper level of atomic sodium and a lower level that is shifted by a strong pump pulse. We also present a

\footnotetext{
*Present address: Department of Physics, University of Texas at Austin, Austin, TX 78712.
}

mathematical model to describe this behavior and compare it with experimental data. As shown in Fig. 1(a), the transition $|1\rangle-|2\rangle$ is driven by a strong resonant pump pulse. Transition $|2\rangle-|3\rangle$ is probed by a coincident weak probe pulse, and we measure fluorescence from level $|4\rangle$ which is populated by spontaneous emission from level $|3\rangle$. The pump pulse produces two time-dependent dressed states [6] with which the probe interacts as shown in Fig. 1(b). For any value of probe detuning that is less than the maximum shift of the dressed state, resonance occurs at two separate times. For a fixed pump-pulse energy, this leads to fringes in the absorption line shape as the probe scans across the $|2\rangle-|3\rangle$ transition.

In our experiment the pump and probe pulses were generated by pulse-amplifying light from single-mode $\mathrm{cw}$ dye lasers. Amplification occurred in chains of dye amplifiers pumped by an injection-seeded neodymium-doped yttrium aluminum garnet (Nd:YAG) laser. Injection seeding assured that the pump light had near-Gaussian time profiles. By carefully adjusting the pump timing and intensity in each stage of the dye chain, the amplified pulses were also made nearly Gaussian. The probe pulse at $568 \mathrm{~nm}$ had an energy up to 10

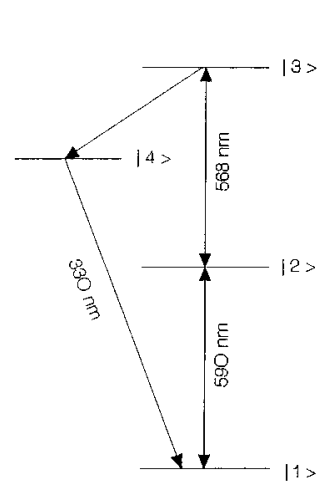

(a)

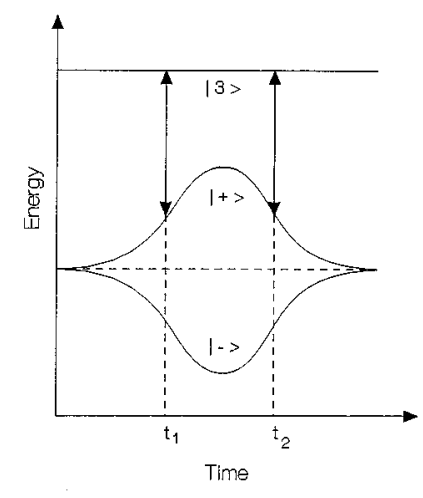

(b)
FIG. 1. (a) The experimental configuration in atomic sodium. Level $|1\rangle$ is $3 S_{1 / 2} F=2, m_{f}=2$, level $|2\rangle$ is $3 P_{3 / 2} F=3, m_{f}=3$, level $|3\rangle$ is $4 D_{5 / 2} F=4, m_{f}=4$, and level $|4\rangle$ is $4 P_{3 / 2} F=3, m_{f}=3$. The double-ended arrows are the pump $(590 \mathrm{~nm})$ and probe $(568 \mathrm{~nm})$ transitions. The single-ended arrows represent spontaneous emission. We observed the 330-nm fluorescence. (b) The time-dependent energy splitting of level $|2\rangle$ and the two paths of absorption for the 568-nm optical field. 
$\mu \mathrm{J}$ and a duration [full width at half maximum (FWHM)] of 4.3 ns. Its measured bandwidth was $155 \pm 10 \mathrm{MHz}$. The pump pulse at $590 \mathrm{~nm}$ had up to $200 \mu \mathrm{J}$ with a duration (FWHM) of $5 \mathrm{~ns}$, and a measured bandwidth of $120 \pm 6$ MHz. These bandwidths, measured using a Fabry-Pérot étalon, are approximately 1.5 times the transform limit. We attribute the excess width to frequency chirps created in the amplification process [7].

The atomic sodium beam was created in the first chamber of a two-chamber vacuum system. Beam-collimating apertures separated the chambers, and the collinear pump and probe beams intersected the atomic beam at a right angle in the second chamber. The measured Doppler shifts are less than $30 \mathrm{MHz}$. Helmholtz coils nulled the magnetic field transverse to the direction of light propagation, and established a field of $7 \times 10^{-4} \mathrm{~T}$ along the propagation direction. Using a portion of the 590-nm cw light with right circular polarization, we optically pumped the sodium atoms so that $95 \%$ of the population in the $3 S_{1 / 2} F=2$ level was in the $m_{F}=+2$ Zeeman level. This light crossed the atomic beam upstream from the pump-probe interaction volume, and the magnetic field maintained the population distribution through the interaction region. The $\mathrm{cw}$ fluorescence signal from the $3 P_{3 / 2} F=3$ level was used to keep the 590-nm laser tuned to exact resonance with the $3 S_{1 / 2} F=2$ to $3 P_{3 / 2} F=3$ transition. The pulsed 590-nm pump light was also right circularly polarized so it interacted only with $3 S_{1 / 2} F=2$, $m_{F}=2$ and $3 P_{3 / 2} F=3, m_{F}=3$ states. The 568-nm pulsed probe light was right circularly polarized so it interacts only with the $3 P_{3 / 2} F=3, m_{f}=3$ and $4 D_{5 / 2} F=4, m_{f}=4$ states. We measured the $4 P$ population by monitoring the $330-\mathrm{nm}$ fluorescence of the $4 P-3 S$ transition.

The 568- and 590-nm beams were spatially filtered, and combined so they exactly overlapped and had the same beam diameter and propagation direction. Their spatial intensity distribution was Gaussian with a diameter (FWHM) of $1 \mathrm{~cm}$. Intensity deviations from the Gaussian were less than $3 \%$. The 330-nm fluorescence was collected perpendicular to the plane of the atomic and light beams. By imaging a 2-mmdiameter region of the interaction volume centered on the peak of the Gaussian spatial profiles, we ensure nearly uniform pump and probe intensities transverse to the detection direction. We verified this by measuring Rabi oscillations [8] separately on the $3 S-3 P$ and $3 P-4 D$ transitions as a function of 590- and 568-nm fluences. These Rabi oscillations also calibrated the pulse areas $\left[\theta=\int_{-\infty}^{\infty} \mu E(t) d t / \hbar\right]$ of the pump and probe fields. For the observation of interference fringes, the pulse areas were fixed at $22 \pi$ and $2 \pi$ for the 590and 568-nm pulses, respectively.

Figure 2 shows the measured fluorescence as a function of tuning of the probe laser. Fringes are clearly evident. This interference effect involves not only interactions separated in time like Ramsey's but also time-dependent eigenstates similar to the examples cited above of atomic collisions and multiphoton transitions. We present here a simplified physical model that describes qualitatively the evolution of phases during the pump-probe interaction. Then we present a more exact numerical model of the interaction and compare its predictions with our experimental results.

For the simplified model we use Schrödinger's equation to find the probability amplitudes of the bare states in inte-

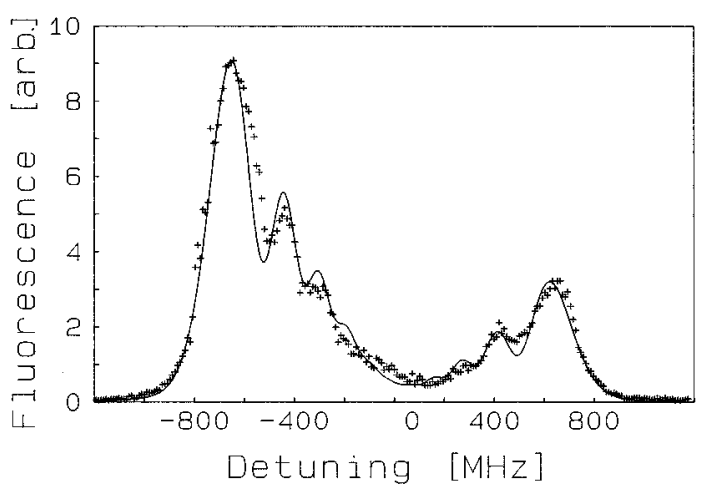

FIG. 2. The solid line is the numerical calculation and the crosses represent the measurement. The numerical calculation is based on measured experimental conditions including the time profiles, the frequency chirps, the spatial intensity profiles, and the pulse areas of the pump and probe as measured by Rabi oscillations.

gral form. The method of stationary phase is then applied to these expressions to pick out the two times when the probe is resonant with one of the dressed states. We then diagonalize the interaction Hamiltonian for the pump alone to find the dressed states [6] and use them to calculate the probe transition amplitudes at these two times. These amplitudes are summed and squared to find the net excitation probability that exhibits the interference fringes.

We start with Schrödinger's equation

$$
i \hbar \frac{\partial \psi(\vec{r}, t)}{\partial t}=\left(H_{0}+V\right) \psi(\vec{r}, t)
$$

where $H_{0}$ is the unperturbed atomic Hamiltonian and $V$ is the interaction Hamiltonian describing the coupling of the atom with the two light fields. We define $\psi(\vec{r}, t)$ as

$$
\psi(\vec{r}, t)=\sum_{n} C_{n}(t)|n(\vec{r})\rangle \exp \left(-i \omega_{n} t\right)
$$

where the $|n(\vec{r})\rangle$ 's are eigenstates of $H_{0}$. We perform a unitary transformation on Eq. (1) to remove the $\exp \left(i \omega_{i j} t\right)$ optical frequencies. The transformation operator is $U(t)$ $=\exp (-i \tilde{H} t / \hbar)$ where

$$
\tilde{H}=\left(\begin{array}{ccc}
\hbar \omega_{590} & 0 & 0 \\
0 & 0 & 0 \\
0 & 0 & \hbar \omega_{568}
\end{array}\right) .
$$

In this representation we have

$$
\frac{\partial C_{n}(t)}{\partial t}=-\frac{i}{\hbar} \sum_{k} C_{k}(t) \mathscr{V}_{n k}
$$

where $\mathscr{V}$ is

$$
\mathscr{V}=\left(\begin{array}{ccc}
0 & \frac{1}{2} \Omega_{590}(t) & 0 \\
\frac{1}{2} \Omega_{590}(t) & 0 & \frac{1}{2} \Omega_{568}(t) \\
0 & \frac{1}{2} \Omega_{568}(t) & -\Delta
\end{array}\right) .
$$

The off-diagonal terms in $\mathscr{V}$ are the time-dependent Rabi frequencies, $\Omega(t)=\vec{\mu} \cdot \overrightarrow{\mathscr{E}}(t) / \hbar$, where $\mu$ is the transition di- 
pole matrix element, and $\vec{E}$ is the optical field defined by $\vec{E}(t)=\overrightarrow{\mathscr{E}}(t)[\exp (-i \omega t)+\exp (i \omega t)] / 2$. The diagonal term $\Delta$ is the detuning of the 568-nm field from the unperturbed resonance. The 590-nm pump is exactly resonant with the $|1\rangle-|2\rangle$ transition. We assume $C_{3}(t) \ll C_{1}(t), \quad C_{2}(t)$ and $\left|C_{1}(t)\right|^{2}+\left|C_{2}(t)\right|^{2} \simeq 1$. This weak-probe approximation is justified because, although the probe area is $2 \pi$, it is resonant with a dressed state only briefly. If all the population is initially in level $|1\rangle$, we find

$$
\begin{aligned}
C_{1}(t)= & \frac{1}{2}\left[\exp \left(\frac{i}{2} \int_{-\infty}^{t} \Omega_{590}\left(t^{\prime}\right) d t^{\prime}\right)\right. \\
& \left.+\exp \left(-\frac{i}{2} \int_{-\infty}^{t} \Omega_{590}\left(t^{\prime}\right) d t^{\prime}\right)\right], \\
C_{2}(t)= & \frac{1}{2}\left[\exp \left(-\frac{i}{2} \int_{-\infty}^{t} \Omega_{590}\left(t^{\prime}\right) d t^{\prime}\right)\right. \\
& \left.-\exp \left(\frac{i}{2} \int_{-\infty}^{t} \Omega_{590}\left(t^{\prime}\right) d t^{\prime}\right)\right] .
\end{aligned}
$$

Using these in Eq. (1), with the assumption that the probe intensity is constant, we find that the probability to be in state $|3\rangle$ is

$$
\begin{aligned}
\left|C_{3}(t)\right|^{2}= & \frac{\left|\Omega_{568}\right|^{2}}{8} \mid \int_{-\infty}^{t} \exp \left(i \Delta \tau-\frac{i}{2} \theta(\tau)\right) d \tau \\
& -\left.\int_{-\infty}^{t} \exp \left(i \Delta \tau+\frac{i}{2} \theta(\tau)\right) d \tau\right|^{2}
\end{aligned}
$$

where $\theta(\tau)=\int_{-\infty}^{\tau} \Omega_{590}\left(t^{\prime}\right) d t^{\prime}$. There are two integrals because level $|2\rangle$ is split into two dressed levels, and a phase evolution is associated with each path. For $\Delta \neq 0$, these integrals can be evaluated using the method of stationary phase [9] to pick out as the primary contributions the points where the derivatives of the functions in the exponents are zero, i.e., $\partial / \partial \tau[\Delta \tau \pm \theta(\tau) / 2]=0$. Assuming a Gaussian pump pulse, $\Omega_{590}(t)=\Omega_{0} \exp \left(-t^{2} / T^{2}\right)$, two points satisfy this condition in the first integral if $0<\Delta<\Omega_{590}(0) / 2$, or two points satisfy it in the second integral if $0>\Delta>-\Omega_{590}(0) / 2$. In the first case, these points correspond to the two times $t_{1}$ and $t_{2}$ when the probe is resonant with the $|-\rangle$ state, while in the second case they correspond to resonance with the $|+\rangle$ state as shown in Fig. 1(b).

Making the further approximation that Eq. (8) can be evaluated by including only the contributions at $t_{1}$ and $t_{2}$, and including only the larger of the two integrals, we use dressed states of the pump-field-atom system to calculate transition amplitudes. The net probability for absorption from either dressed state $|+\rangle$ or $|-\rangle$ is the square of the sum of these two amplitudes. The dressed-state eigenenergies and eigenstates are $\lambda_{ \pm}= \pm \Omega_{590}(t) / 2$ and $| \pm\rangle=1 / \sqrt{2}(|1\rangle \pm|2\rangle)$ so the probability may be written as

$$
\mathscr{P}=\left|\left\langle 3\left|\vec{E}_{568} \cdot \vec{\mu}_{23}\right| \pm\right\rangle_{t_{1}}+\left\langle 3\left|\vec{E}_{568} \cdot \vec{\mu}_{23}\right| \pm\right\rangle_{t_{2}} e^{i \beta}\right|^{2}
$$

where the phase difference $\beta$ is given by

$$
\beta=\int_{t_{1}}^{t_{2}}\left[\Delta \pm \frac{1}{2} \Omega_{590}(t)\right] d t
$$

For synchronous symmetric pump and probe pulses, the probability can be expressed as

$$
\mathscr{P}=\left|\left\langle 3\left|\vec{E}_{568} \cdot \vec{\mu}_{23}\right| 2\right\rangle\right|^{2}(1+\cos \beta)
$$

for resonance with either dressed state. Note that the relative phase of the two contributions, and thus the phase of the interference, depends on $\int_{t_{1}}^{t_{2}} \Omega_{590}\left(t^{\prime}\right) d t^{\prime} / 2$ as well as $\Delta\left(t_{2}-t_{1}\right)$. This is in contrast to the Ramsey phase which is equal to $\Delta\left(t_{2}-t_{1}\right)$. Our result is similar to that derived by Aharonov and Anandan [10] for time-varying energy levels. They characterize the portion of the phase that is due to $\int_{t_{1}}^{t_{2}} \Omega_{590}\left(t^{\prime}\right) d t^{\prime} / 2$ as a geometrical phase.

The simple theory just presented gives a physical picture of this interaction and correctly predicts the positions of the fringes, but it does not accurately predict the amplitudes of the fringes. We find in evaluating Eq. (8) numerically that the interaction cannot be accurately approximated as occurring only at two separate times. The level $|3\rangle$ population actually undergoes large oscillations in the time interval between $t_{1}$ and $t_{2}$. The other problem with the simplified treatment is that it ignores spontaneous emission from levels $|2\rangle$ and $|3\rangle$.

To describe the experiment more accurately, we numerically integrate the equation of motion for the density matrix [11]:

$$
\frac{\partial \rho}{\partial t}=\frac{-i}{\hbar}[\mathscr{V}, \rho]-\frac{1}{2}(\Gamma \rho+\rho \Gamma) .
$$

Here $\Gamma$ is the diagonal matrix of spontaneous decay rates. Except for frequency chirps, we use only directly measured experimental parameters in our model. In order to match the experiment we had to make some assumptions about the nature of the chirps on the pump and probe beams. As mentioned above, we found using a Fabry-Pérot étalon that the pump and probe bandwidths were approximately 1.5 times the transform limit. We also measured Rabi oscillations on each transition, and found that the modulation depths were

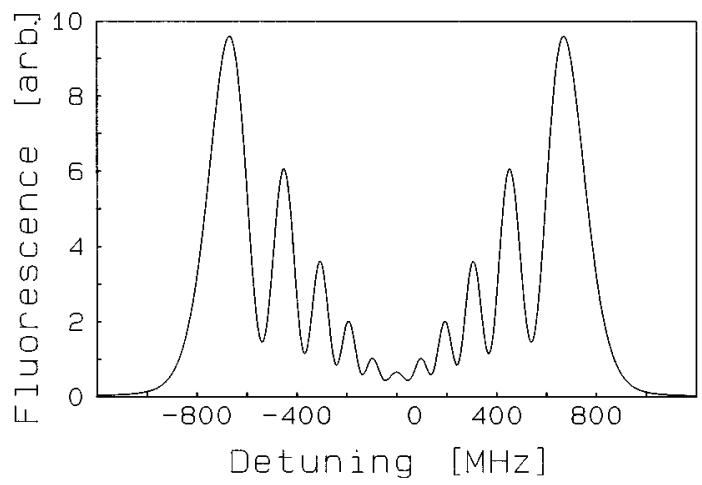

FIG. 3. This is a numerical simulation where the pump and probe fields are bandwidth-limited 5-ns Gaussian pulses that are simultaneous. The pulse areas are the same as the experimental values. Compared with Fig. 2 there is a substantial improvement in the fringe contrast. 
less than expected for transform-limited bandwidths. By assuming that the excess bandwidth was due to a linear chirp we were able to model the measured Rabi oscillations accurately. A linear chirp is also consistent with the observations of Melikechi, Gangopadhyay, and Eyler [7] who measured chirps for similar pulse amplification in dyes. The Rabi oscillations are not sensitive to the directions of the chirps, but the direction of the pump chirp strongly influences the relative heights of the red and blue sidebands in Fig. 2. According to our numerical model, if the chirp is blue to red, the red sideband should be taller. For a red-to-blue chirp, the reverse is true. Using a linear blue-to-red chirp consistent with the Fabry-Pérot and Rabi oscillation measurements, we get excellent agreement with the line-shape experiment as shown in Fig. 2.

The fringes, although clearly observable, are muted by the chirps and by the fact that the probe pulse is slightly shorter than and not quite synchronous with the pump pulse. The contrast of the fringes could be dramatically improved if the pump and probe were exactly simultaneous, transformlimited pulses, and if the probe were slightly longer than the pump. Figure 3 shows a calculation of a line shape for the same pulse areas as the experiment but with no chirp and with pulse durations of $5 \mathrm{~ns}$.

In conclusion, we have experimentally and theoretically demonstrated a quantum interference effect that is related to time-separated interactions. The acquired phase of the atom is caused by the evolution of the dressed states created by the strong pump field.

S.R.W. would like to thank Joe Alford for discussions of interference in atomic collisions, and Jon Dowling, Aaron Manka, Darrell Armstrong, and Hans Briegel for helpful discussions. This work was performed at Sandia National Laboratories under DOE Contract No. DE-AC04-94AL85000. M.O.S. would like to thank the ONR, the Welch Foundation, and the TARP for their support.
[1] N. F. Ramsey, Phys. Rev. 76, 996 (1949).

[2] M. M. Salour, Rev. Mod. Phys. 50, 667 (1978).

[3] A. Royer, Phys. Rev. A 4, 499 (1971).

[4] M. C. Baruch and T. F. Gallagher, Phys. Rev. Lett. 68, 3515 (1992).

[5] R. R. Jones, Phys. Rev. Lett. 74, 1091 (1995).

[6] C. Cohen-Tannoudjii, J. Dupont-Roc, and G. Grynberg, AtomPhoton Interactions (Wiley, New York, 1992).
[7] N. Melikechi, S. Gangopadhyay, and E. E. Eyler, J. Opt. Soc. Am. B 11, 2402 (1994).

[8] A. V. Smith and T. D. Raymond, Phys. Rev. A 45, 4688 (1992).

[9] E. T. Copson, Asymptotic Expansions (Cambridge University Press, Cambridge, England, 1965).

[10] Y. Aharonov and J. Anandan, Phys. Rev. Lett. 58, 1593 (1987).

[11] M. Sargent, M. O. Scully, and W. E. Lamb, Laser Physics (Addison-Wesley, Reading, MA, 1974). 